\title{
ATLAS Forward Proton detectors: first experience with data
}

\author{
James L. Pinfold ${ }^{* \dagger}$ \\ University of Alberta \\ E-mail: jpinfoldeualberta.ca
}

The aim of the ATLAS Forward Proton (AFP) detector system is the measurement of protons scattered diffractively or electromagnetically at very small angles. The first arm of the system was installed last year and AFP took data in several commissioning and physics runs. The installation of the second arm has just been completed. This will allow measurements of processes with two forward protons: central diffraction, exclusive production, and two-photon processes.

The European Physical Society Conference on High Energy Physics

5-12 July

Venice, Italy

* Speaker.

${ }^{\dagger}$ For the AFP Group. 


\section{Introduction}

The ATLAS Forward Proton (AFP) project promises a significant extension to the physics reach of ATLAS by tagging and measuring the momentum and emission angle of very forward protons. The accompanying high resolution Time of Flight (ToF) system enables us to suppress pileup background and operate at standard (high) luminosity. AFP enables the observation and measurement of a range of processes where one or both protons remain intact which otherwise would be difficult or impossible to study. Such processes are typically associated with elastic and diffractive scattering, where the proton radiates a virtual colourless Pomeron, which can be thought of as a non-perturbative collection of soft gluons. Diffractive processes can also be measured in the context of heavy-ion collisions, and open up a new program of study in this field.

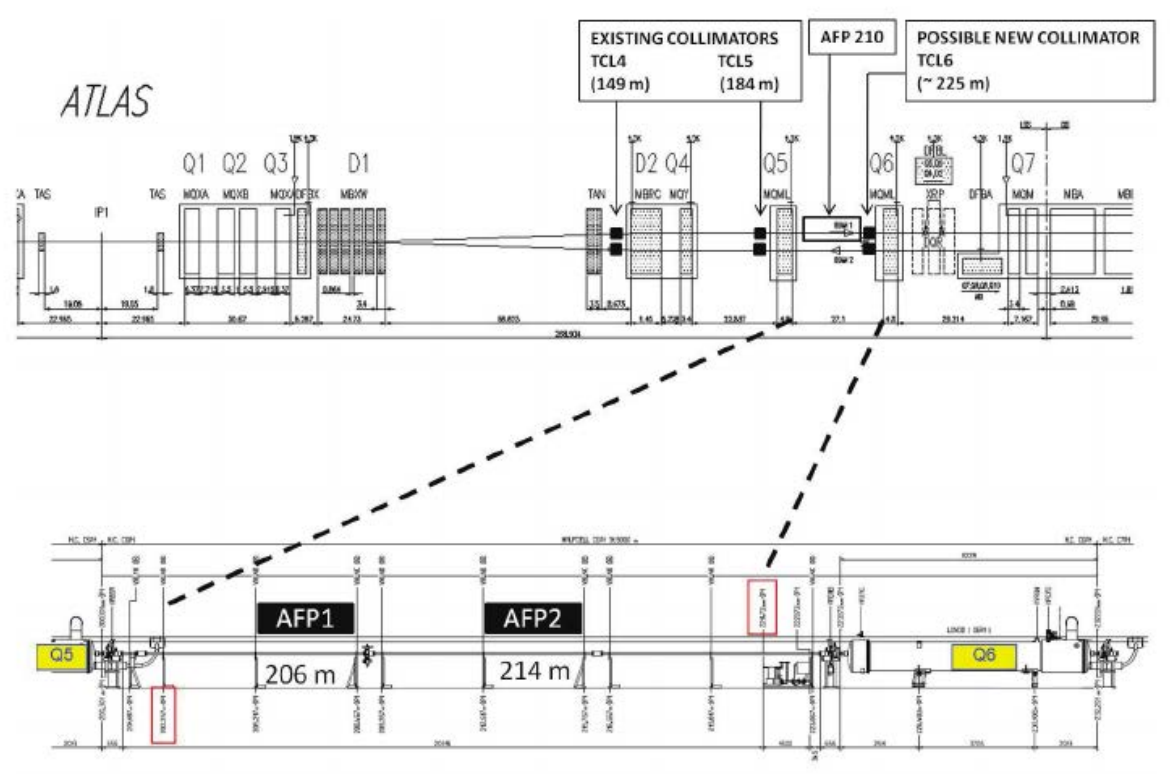

Figure 1: The positions of one arm of AFP detectors, the other arm is positioned symmetrically wrt to the arm shown here.

Forward proton tagging allows the LHC to function as a energy-tuneable gluon-gluon, photonphoton or photon-gluon collider. In Central Exclusive Production, recently observed by CDF [1], the entire momentum loss of the protons goes into the creation of the central system, providing a particularly clean environment to search for and characterize any new resonance. Since the central system is produced in a JPC $=0^{++}$state, backgrounds from di-quark production are suppressed, and the mere observation of a resonance determines its quantum numbers.

An example of a prime topic in AFP's physics program is the two-photon production of Wpairs which has a relatively large cross section - anomalous couplings should be measurable in this process during normal high luminosity data taking, or at least limits can be put upon them. ATLAS [2] and CMS [3] have already improved existing anomalous coupling limits by about two orders of magnitude. Proton tagging will allow yet another two orders improvement. 


\section{The AFP Detector}

The ATLAS Forward Proton detectors aim to measure protons emitted from a central interaction in the very forward directions. Protons suffering a moderate energy loss due to an interaction, and emitted at $\mu$-rad angles wrt the beams, will remain inside the beam pipe but separate from the beam axis due to the accumulated dispersion in the beam elements. The AFP detector consists of 2 stations at $\pm 205 \& \pm 217 \mathrm{~m}$ from the IP are housed in horizontal Roman Pots. The positioning of the AFP detectors on the LHC beamline is depicted in Fig. 1.

Near stations house 3D-Si pixel tracking detectors that tag and measure momentum and emission angle of forward protons. Far stations also have Time of Flight (ToF) counters to identify the origin of protons in longitudinal direction with $\Delta \mathrm{z}=2 \mathrm{~mm}$, if the time resolution of ToF is $10 \mathrm{ps}$. A 3D depiction of a far detector is shown in Fig. 2.

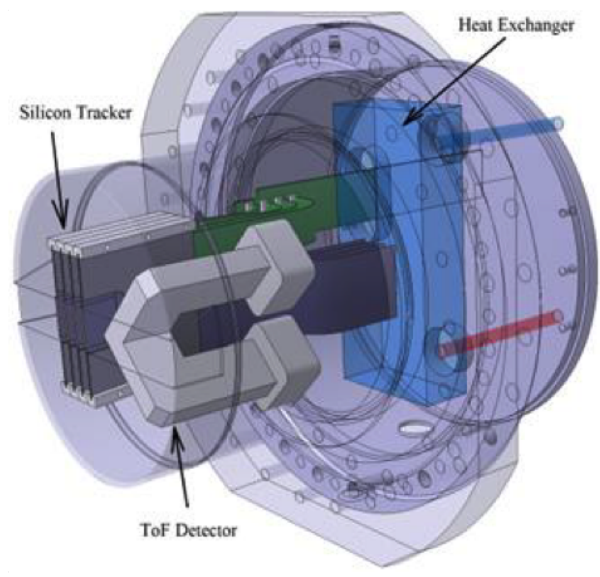

Figure 2: A depiction of a far station of the Roman Pot based AFP detector.

At around $200 \mathrm{~m}$ from the ATLAS Interaction Point (IP) the deflected protons will be sufficiently separated from the nominal beam orbit so that they can be intercepted by the AFP Roman pots inserted into the beam pipe aperture. The deflection of the proton depends on the magnitude of the energy loss suffered, and also on the emission angle at the IP. The criteria for the selection of the AFP positions are: availability of space; and, sufficient integral dispersion to make interception and measurement viable.

Two measurements are required to characterize the emitted proton fully - the transverse position and the local angles. Together, these measurement give the proton fractional energy loss $\xi$ ( $\xi=1-E_{\text {proton }} / E_{\text {beam }}$ ) and the four-momentum transfer squared $t$, as a function of the emission angle $\theta$. Combined with the intervening beam optics, these variables fully determine the emitted proton's momentum four vector.

\subsection{The Tracking Detectors}

The tracking detectors together with LHC magnets measure the momentum of the forward scattered protons. A position resolution of $15 \mu \mathrm{m}$ in the tracking detector is required to meet the physics aims of AFP. Radiation hardness is crucial for AFP to be able to operate at luminosities up 
to $\left.5 \times 10^{15} \mathrm{p} / \mathrm{cm}^{2} / 100 \mathrm{fb}^{1}\right)$. In order to get as close to the beam as possible (2-3 mm) the inactive edge is small ( $\sim 200 \mu \mathrm{m}$ ). In each station we have 4 pixel layers with $336 \times 80$ pixels (each $50 \times$ $250 \mu \mathrm{m}^{2}$ ). The 3D-Si pixel detector technology utilized here is the same as that used in ATLAS's IBL. A detailed description of the AFP tracking detectors is provided elsewhere [4].

\subsection{The Timing Detectors}

An high-resolution Time-of-Flight ( $\mathrm{ToF}$ ) detector will be needed for the reduction of pile-up backgrounds at high- $\mu$. The AFP ToF detectors are a modified version of the original Quartic detector, proposed by FP420 [5]. The Quartic detector consisted of straight synthetic quartz Qbars positioned at the Cherenkov angle with respect to the proton flight direction, functioning both as a radiator producing Cerenkov light and as a light guide that transmits the light to the phototube. The AFP ToF detector is very similar in concept. However, space constraints imposed by the cylindrical Roman pot housing require that a hybrid "L"-shaped "LQbar" solution was adopted which combines the best features of earlier designs. The Cerenkov light generated in the quartz radiators travels up the bars and is converted to a signal by a specialized Photonis Mini-planicon Microchannel-Plate Photomultipliers [6]. The ToF detector timing resolution achieved to date is $\sigma_{T} \sim 20 \mathrm{ps}$. Also, the ToF detector has sufficient granularity to discern multiple incoming protons. More details are given elsewhere [4].

\section{Status of the AFP Deployment}

The installation of both arms of AFP was completed in 2016/2017. AFP has passed LHC qualification and is integrated into the ATLAS trigger and DAQ system. Commissioning runs have taken place in 2016 under low pileup $(\mu<0.3$, where $\mu$ is the average number of interactions per beam crossing) and high luminosity ( $\mu_{\max } \sim 35$ and $3 \rightarrow 600$ bunches). Commissioning runs continue in 2017 with the full detector.

\section{References}

[1] For example: T. Aaltonen et al., Phys. Rev. Lett. 102242001 (2009).

[2] G. Aad et al., ATLAS Collaboration, Phys. Rec. Lett. 113, 141804 (2014) G. Aad et al., Phys. Rev. Lett. 115, 031802 (2015).

[3] S. Chatrchyan et al., CMS Collaboration, J. High Energy Phys. 07, 116 (2013); S. Chatrchyan et al., CMS Collaboration, Phys. Rev. D90, 032008 (2014).

[4] L. Adamczyk et al., Technical Design Report for the ATLAS Forward Proton Detector May 20, 2015. CERN-LHCC-2015-009, ATLAS-TDR-024.

[5] http://optics.heraeus-quarzglas.com. Suprasil, Premium Synthetic Quartz Glass for deep UV Applications by Heraus Quarzglass.

[6] Photonis Corporation, www.photonis.com. 